\title{
A Survey of Semisimple Algebras in Algebraic Combinatorics
}

\author{
Allen Herman* \\ Received February 8, 2020. \\ Revised April 30, 2020
}

\begin{abstract}
This is a survey of semisimple algebras of current interest in algebraic combinatorics, with a focus on questions which we feel will be new and interesting to experts in group algebras, integral representation theory, and computational algebra. The algebras arise primarily in two families: coherent algebras and subconstituent (aka. Terwilliger) algebras. Coherent algebras are subalgebras of full matrix algebras having a basis of 01-matrices satisfying the conditions that it be transpose-closed, sum to the all 1's matrix, and contain a subset $\Delta$ that sums to the identity matrix. The special case when $\Delta$ is a singleton is the important case of an adjacency algebra of a finite association scheme. A Terwilliger algebra is a semisimple extension of a coherent algebra by a set of diagonal 01-matrices determined canonically from its basis elements and a choice of row. We will survey the current state of knowledge of the complex, real, rational, modular, and integral representation theory of these semisimple algebras, indicate their connections with other areas of mathematics, and present several open questions.
\end{abstract}

Key words : Coherent algebras, coherent configurations, association schemes, adjacency algebras, Terwilliger algebras, table algebras, based rings, Schur rings, units, zeta functions.

AMS Classification: Primary: 05E30; Secondary: 05E10, 05E15, 11S45, 16G30, 16S99, 16U60, 20C10, 20C15, 20C20.

*The author's work has been supported by NSERC Discovery Grant. 


\section{The algebras}

We will first introduce all of the semisimple algebras we wish survey, give some motivation for their study, and present some of their basic properties.

Definition 1. [35] A coherent algebra $(\mathrm{CA})$ is a subalgebra $\mathbb{C B}$ of $M_{n}(\mathbb{C})$ defined by a special basis $\mathbf{B}$, which is a collection of non-overlapping 01-matrices that

(1) is a closed set under the transpose,

(2) sums to $J$, the $n \times n$ all 1 's matrix, and

(3) contains a subset $\Delta$ of diagonal matrices summing $I$, the identity matrix.

The standard basis $\mathbf{B}$ of a $\mathrm{CA}$ in $M_{n}(\mathbb{C})$ is precisely the set of adjacency matrices of a coherent configuration (CC) of order $n$ and rank $r=|B|$. Each 01matrix $b$ in our basis is viewed as the adjacency matrix of a relation $R_{b}$ on the set of vertices $X=\{1, \ldots, n\}$, with the entry in the $x y$-position of the matrix $b$ being a 1 if and only if $x$ and $y$ are related by $R_{b}$, and otherwise it is 0 . The set of diagonal basis elements are called fibres of the CC. When $\Delta=\{I\}$, the $\mathbf{B}$ is the set of adjacency matrices of an association scheme (AS), and the algebra $\mathbb{C B}$ is the adjacency algebra of the AS, often called the Bose-Mesner algebra. The ASs correspond to homogeneous $\mathrm{CCs}$, the CCs with only one fibre.

If $\mathbf{B}=\left\{b_{0}, b_{1}, \ldots, b_{r-1}\right\}$ is the set of adjacency matrices of a $\mathrm{CC}$, then any element $a$ of the $\mathbf{C A} \mathbb{C B}$ is a $\mathbb{C}$-linear combination of $\mathbf{B}$, so it can be written as $a=\sum_{i=0}^{r-1} a_{i} b_{i}$. We can regard the CA as an algebra with skew-linear involution determined by $\left(\sum_{i} a_{i} b_{i}\right)^{*}=\sum_{i} \overline{a_{i}} b_{i}^{\top}$. The fact that $\mathbf{B}^{\top}=\mathbf{B}$ implies that $\mathbb{C B}$ is a self-adjoint subalgebra of $M_{n}(\mathbb{C})$ and is hence semisimple.

(1.1) Examples. To see examples, we use the fact that an AS or CC can be illustrated by its basic matrix.

(1.1.1). Here we give the basic matrix of an AS with standard basis $\mathbf{B}=$ 
$\left\{b_{0}, b_{1}, \ldots, b_{7}\right\}:$

$$
\sum_{i=0}^{d} i b_{i}=\left[\begin{array}{cccccccccccc}
0 & 1 & 2 & 3 & 4 & 4 & 5 & 5 & 6 & 6 & 7 & 7 \\
1 & 0 & 3 & 2 & 4 & 4 & 5 & 5 & 6 & 6 & 7 & 7 \\
2 & 3 & 0 & 1 & 6 & 6 & 7 & 7 & 4 & 4 & 5 & 5 \\
3 & 2 & 1 & 0 & 6 & 6 & 7 & 7 & 4 & 4 & 5 & 5 \\
4 & 4 & 7 & 7 & 0 & 1 & 6 & 6 & 5 & 5 & 2 & 3 \\
4 & 4 & 7 & 7 & 1 & 0 & 6 & 6 & 5 & 5 & 3 & 2 \\
5 & 5 & 6 & 6 & 7 & 7 & 0 & 1 & 2 & 3 & 4 & 4 \\
5 & 5 & 6 & 6 & 7 & 7 & 1 & 0 & 3 & 2 & 4 & 4 \\
7 & 7 & 4 & 4 & 5 & 5 & 2 & 3 & 0 & 1 & 6 & 6 \\
7 & 7 & 4 & 4 & 5 & 5 & 3 & 2 & 1 & 0 & 6 & 6 \\
6 & 6 & 5 & 5 & 2 & 3 & 4 & 4 & 7 & 7 & 0 & 1 \\
6 & 6 & 5 & 5 & 3 & 2 & 4 & 4 & 7 & 7 & 1 & 0
\end{array}\right]
$$

One of the most important properties of ASs is that each $b_{i} \in \mathbf{B}$ has the same number of 1's in each row and column, namely the coefficient of $I=b_{0}$ in the product $b_{i} b_{i}^{\top}$. This positive integer is called the valency of $b_{i}$, which we will denote by $\delta\left(b_{i}\right)$. Observe that this value is the eigenvalue of $b_{i}$ relative to the all 1's eigenvector, which is common to all the $b_{i}$. This means the valency extends to a one-dimensional representation of $\mathbb{C B}$ that takes positive values on elements of $\mathbf{B}$. Orthogonality of irreducible characters of the semisimple algebra with involution $\mathbb{C B}$ tells us the valency map is the unique irreducible character with this property.

(1.1.2). CCs that are not ASs also have a basic matrix, but because there is more than one fiber this basic matrix will have more than one distinct entry on its diagonal. Here is an example of a basic matrix of a CC of order 10 and $\Delta=\left\{b_{0}, b_{6}\right\}$ :

$$
\sum_{i=0}^{d} i b_{i}=\left[\begin{array}{cccccccccc}
0 & 1 & 2 & 3 & 2 & 3 & 2 & 3 & 2 & 3 \\
1 & 0 & 3 & 2 & 3 & 2 & 3 & 2 & 3 & 2 \\
4 & 5 & 6 & 7 & 8 & 7 & 8 & 7 & 8 & 7 \\
5 & 4 & 7 & 6 & 7 & 8 & 7 & 8 & 7 & 8 \\
4 & 5 & 8 & 7 & 6 & 7 & 8 & 7 & 8 & 7 \\
5 & 4 & 7 & 8 & 7 & 6 & 7 & 8 & 7 & 8 \\
4 & 5 & 8 & 7 & 8 & 7 & 6 & 7 & 8 & 7 \\
5 & 4 & 7 & 8 & 7 & 8 & 7 & 6 & 7 & 8 \\
4 & 5 & 8 & 7 & 8 & 7 & 8 & 7 & 6 & 7 \\
5 & 4 & 7 & 8 & 7 & 8 & 7 & 8 & 7 & 6
\end{array}\right]
$$

The $\sum \Delta=I, \sum \mathbf{B}=J$, and $\mathbf{B}^{\top}=\mathbf{B}$ properties are clear from the basic matrix presentation of the $\mathrm{CC}$, the property $\mathbf{B}^{2} \subset \mathbb{N B}$ which says $\mathbf{B}$ is the basis of an algebra with nonnegative structure constants is the only one that needs to be 
carefully checked. Note that the fibres naturally produce a block decomposition structure for the basic matrices in which 1's will only appear in one block. The subset of basis elements appearing in the same block along the main diagonal will form an AS in that block.

(1.1.3). By means of its left regular representation, a finite group $G$ of order $n$ produces an AS whose set of vertices is $G$, so both the order and the rank of this AS is $n$. These are the only ASs for which the valency of every element is 1 , so they are referred to as the thin ASs. Note that the valency map for a thin AS corresponds to the augmentation map of the group algebra for the corresponding group. For example, here is a (possible) basic matrix for the quaternion group $Q_{8}$ :

$$
\sum_{i=0}^{7} i b_{i}=\left[\begin{array}{cccccccc}
0 & 1 & 2 & 3 & 4 & 5 & 6 & 7 \\
1 & 0 & 3 & 2 & 5 & 4 & 7 & 6 \\
3 & 2 & 0 & 1 & 6 & 7 & 5 & 4 \\
2 & 3 & 1 & 0 & 7 & 6 & 4 & 5 \\
5 & 4 & 7 & 6 & 0 & 1 & 2 & 3 \\
4 & 5 & 6 & 7 & 1 & 0 & 3 & 2 \\
7 & 6 & 4 & 5 & 3 & 2 & 0 & 1 \\
6 & 7 & 5 & 4 & 2 & 3 & 1 & 0
\end{array}\right]
$$

(The reader should verify the identity $i j=k=-i j$ corresponds to $b_{2} b_{4}=b_{7}=$ $b_{5} b_{2}$.)

(1.1.4). The action of a finite group $G$ a finite set $X$ produces a special type of CC called a two-orbit configuration, whose relations are simply the orbits of $G$ on $X \times X$. This CC has one fiber for each orbit of $G$ on $X$, so it will be an AS precisely when the action of $G$ on $X$ is transitive. An AS that coincides with the two-orbit CC for a transitive group action is known as a Schurian AS. The adjacency algebras of Schurian association schemes are isomorphic to "double coset" Hecke algebras $e_{H} \mathbb{C} G e_{H}$, where $e_{H}$ is the idempotent $\frac{1}{|H|} \sum_{h \in H} h$, and $H$ denotes the stabilizer of any fixed base point $x \in X$.

(1.1.5). Other important families of ASs are generated by graphs, and indeed this viewpoint is used very often in algebraic graph theory. For any directed graph $\Gamma$ with finite vertex set $X=\{1, \ldots, n\}$, we let $A_{\Gamma}$ be the 01 -matrix whose $(i, j)$-entry is a 1 if and only if $\Gamma$ has an edge from vertex $i$ to vertex $j$. Let $J_{n}$ be the $n \times n$ matrix for which every entry is a 1 , and let $I_{n}$ be the $n \times n$ identity matrix, which correspond to the graph on $X$ whose only edges consist of a loop at every $i \in X$. The adjacency matrix of the (loopless) complete undirected graph $J_{n}-I_{n}$ generates the unique rank 2 AS $K_{n}=\left\{I_{n}, J_{n}-I_{n}\right\}$ of order $n$. The adjacency matrix $A$ of a strongly regular graph (SRG) with $n$ vertices produces a rank 3 symmetric AS $\left\{I_{n}, A, A^{c}:=J_{n}-I_{n}-A\right\}$ of order $n$ - in fact one can take this as a definition for an 
SRG. The structure constants of this AS are the parameters of the strongly regular graph: if $A A^{c}=u A+v A^{c}$ and $\delta(A)=k$, then $A^{2}=k I+(k-u-1) A+(k-v) A^{c}$, and these paramters satisfy $k(n-k-1)=k u+(n-k-1) v$. Similarly, the adjacency matrix $A_{1}$ of a distance regular graph (DRG) on $n$ vertices with distance matrices $A_{0}, A_{1}, A_{2}, \ldots, A_{d}$ is precisely defined by the fact that it produces a symmetric AS with adjacency matrices $\left\{A_{0}, A_{1}, \ldots, A_{d}\right\}$ of order $n$ and rank $d+1$. This type of AS is called $P$-polynomial or metric. The adjacency matrix $A$ of a doubly regular tournament (DRT) of order $n=4 u+3$ produces an asymmetric rank $3 \mathrm{AS}$ $\left\{I_{n}, A, A^{\top}\right\}$, its parameters are $A A^{\top}=(2 u+1) I+u A+u A^{\top}, A^{2}=u A+(u+1) A^{\top}$. The converse is also true, any asymmetric rank 3 AS is generated by a DRT. SRGs, DRGs, and DRTs are a very active topic in algebraic graph theory, for this context and its strong connections we refer the reader to the monographs [13] and [7]. Any AS of order $n$ corresponds to an algebraic decomposition of the complete directed graph $K_{n}$ into regular graphs and pairs of totally oriented regular digraphs.

\section{(1.2) Properties.}

(1.2.1) Connection with group rings. Let $S_{n}$ be the symmetric group, and let $\Phi: S_{n} \rightarrow M_{n}(\mathbb{Z})$ be its Coxeter representation which maps each $\sigma \in S_{n}$ to its corresponding permutation matrix which acts on the set of elementary basis vectors $\left\{e_{1}, \ldots, e_{n}\right\}$ by $P_{\sigma}\left(e_{i}\right)=e_{\sigma(i)}$ for $i=1, \ldots, n$.

Proposition 2. Suppose $(X, S)$ is an association scheme of order $n$. Then the integral adjacency algebra $\mathbb{Z} S$ is isomorphic to a subring of $\Phi\left(\mathbb{Z} S_{n}\right)$.

Proof. Suppose $S=\left\{b_{0}, b_{1}, \ldots, b_{r-1}\right\}$ is the set of adjacency matrices of an AS of order $n$. $b_{0}=P_{(1)}$ corresponds to the identity permutation. By the valency condition, each $b_{i} \neq b_{0}$ is a doubly stochastic 01-matrix. By an old result of Birkhoff [15], these $b_{i}$ 's are sums of (non-overlapping) permutation matrices, all of which have to be derangements, because the diagonal entries of $b_{i}$ are all 0 . So the standard representation of $\mathbb{Z} S$ maps it bijectively onto a subring of $\Phi\left(\mathbb{Z} S_{n}\right)$.

(1.2.2) The pseudo-inverse condition. Let $\mathbf{B}=\left\{b_{0}=I_{n}, b_{1}, \ldots, b_{r-1}\right\}$ be the standard basis of an AS. Let $\left\{\lambda_{i j k}\right\}$ be the structure constants relative to $\mathbf{B}$, so

$$
\forall b_{i}, b_{j} \in \mathbf{B}, \quad b_{i} b_{j}=\sum_{k} \lambda_{i j k} b_{k} .
$$

Then $\forall b_{i} \in \mathbf{B}, \exists ! b_{j} \in \mathbf{B}$ such that $\lambda_{i j 0} \neq 0$. (For an AS $b_{j}=b_{i}^{\top}$.)

If we write this $b_{j}$ as $b_{i^{*}}$ for all $i$, then we have

$$
\lambda_{i j 0} \neq 0 \Longleftrightarrow j=i^{*} \text {, and } \lambda_{i^{*} i 0}=\lambda_{i i^{*} 0}>0 \text {. }
$$


This condition is known as the pseudo-inverse condition on the basis B. A table algebra $(\mathrm{TA})$ is an associative algebra with involution $*$ over $\mathbb{C}$ that has a $*$ invariant basis $\mathbf{B}=\left\{b_{0}=1, b_{1}, \ldots, b_{r-1}\right\}$ with nonnegative real structure constants that satisfies the pseudo-inverse condition. Table algebras (abbr. TAs) share many properties in common with adjacency algebras of ASs, this is primarily due to the existence of a degree map that plays the role of the valency/augmentation map. The existence of the degree map is a consequence of Perron-Frobenius theory (see [2]). If the structure constants are only required to be real instead of nonnegative real in the TA definition, one obtains Blau's definition of reality-based algebras (see [9]). Algebraically there is a big price for doing this, because one may no longer have a map to play the role of the augmentation/valency map. For a generalization in the direction of CAs, one can replace the condition that the distinguished basis $\mathbf{B}$ contains 1 with the existence of a set of fibers $\Delta \subset \mathbf{B}$ that sums to 1 , and suitably modify the pseudo-inverse condition to: $\forall i, \exists ! j, k$ such that $b_{k} \in \Delta$ and $\lambda_{i j k}=\lambda_{j i k}>0$. This gives Lusztig's definition of based algebras [41].

The existence of a degree map implies that any TA basis has a unique rescaling by positive scalars so that $\delta\left(b_{i}\right)=\lambda_{i i^{*} 0}$ for all $i$. This rescaled basis is called the standard basis of the TA. Alternatively, one could rescale to obtain a transitional basis, which has the property that $\delta\left(b_{i}\right)=1$ for all $i$. When the transitional basis of a TA has integral structure constants, the free $\mathbb{Z}$-module of this basis produces a fusion ring. (Fusion rings are the finite rank versions of fusion categories, a topic currently receiving a lot of attention in representation theory - see [36].)

(1.2.3) The AS feasibility problem for SITAs. If the structure constants relative to the standard basis of a TA are all nonnegative integers, we refer to the TA as a standard integral table algebra (SITA). Adjacency algebras of ASs are special cases of SITAs. For these modifications of the CA or Adjacency algebra of AS definitions, integrality of structure constants is a strong restriction. An even stronger condition is the feasibility of a SITA or based algebra as the adjacency algebra of an AS or CC.

Question 1. (Feasibility Problem) Which SITAs are represented by ASs? That is, if there exists a SITA $\mathbf{B}=\left\{b_{0}=1, b_{1}, \ldots, b_{r-1}\right\}$ with a given collection of nonnegative integer structure constants, is there a transpose-closed set of $n \times n$ 01-matrices $S$ that sums to $J_{n}$ and is a basis of an $r$-dimensional subalgebra of $M_{n}(\mathbb{C})$ with the same structure constants?

The general feasibility problem would be to find necessary and sufficient conditions for a table algebra basis $\mathbf{B}$ to be feasible as an AS. Bangteng Xu, using ideas concerning hypergroup actions, showed the feasibility condition is equivalent to the condition that the SITA $(A, \mathbf{B})$ act "maximally and irreducibly" on a set of 
order $n$. [53]. While checking this condition is computationally difficult, there are some practical necessary conditions:

- The multiplicity of each irreducible character of $\mathbb{C B}$ in the standard feasible trace of the SITA must be positive integers. This is equivalent to the existence of an $n$-dimensional standard representation $\Phi$ of the table algebra, and reduces the problem to realizing the elements of $\Phi(\mathbf{B})$ as a set of transposed-closed 01-matrices that sum to 1 .

- The structure constants must satisfy the "handshaking lemma": if $b_{j}^{*}=b_{j}$, then for all $b_{i} \in \mathbf{B}$ with $\delta\left(b_{i}\right)>1, \lambda_{i} j i \delta\left(b_{i}\right)$ must be even (for a proof see [31, Lemma 7]).

- The spectrum of each $\Phi\left(b_{i}\right)$ must satisfy all necessary restrictions to be the spectrum of a graph - this is a mainstream topic for researchers in algebraic graph theory. (For the latest computational support for this problem see [48].)

Here are two instances of the feasibility problem that are more likely to be accessible by current methods:

Question 2. (a) Is there a symmetric $A S$ of rank 3 and order 65 with $b_{1}^{2}=$ $32 b_{0}+15 b_{1}+16 b_{2}$ ?

(This is the smallest SRG not known to exist, there are many others, see [12] for an extensive list of symmetric P-polynomial SITAs defined by their intersection arrays, that are not known to exist. The missing Moore graph (a 57-regular SRG on 3250 vertices) is the most famous open case of this sort of feasibility problem.)

(b) [29] Is there a noncommutative AS of rank 7 with exactly four asymmetric relations? (The construction of SITAs of this type in [29] does not produce ASs.)

(1.3) Classifications of CCs and ASs. An effort to classify CCs and ASs using computers has been ongoing since the 1990s. Here we give the current state of these classification efforts:

- CCs have been classified using a computer up to order 15 by Ziv-Av [38]. In this paper Klin and Ziv-Av also describe the smallest CC, with order 14, that is not a two-orbit configuration.

- Hanaki and Miyamoto classified all ASs of orders $n \leq 30$, and $n=32,33$, and 34 [27], and gave partial classifications for many other orders. Their work reduced the classification of ASs of order 31 to the classification of DRTs of order 31, which was just completed in November 2019 by Hanaki, Kharaghani, Mohammadian, and Tayfeh-Rezaie (see [26]). For order 35, DRTs and rank 4 ASs remain unclassified to date. The smallest non-Schurian AS corresponds to a DRT of order 15 . 
SITAs of ranks 3 and 4 have been classified, but due to the feasibility problem it is hard to say which ones correspond to ASs. Rank 3 SITAs are classified by the above parameter restrictions on SRGs and DRTs. A similar classification of parameters can be accomplished for symmetric or asymmetric SITAs of rank 4 . For ranks 5 and higher, certain types of ASs can be ruled out by character theory restrictions:

- Noncommutative rank 5 ASs do not exist [55] - neither do noncommutative rank 5 SITAs [32].

- There are no noncommutative rank 7 ASs with 6 asymmetric relations [29]. All examples known to the authour have 2 asymmetric relations, this is the inspiration for Question $2 \mathrm{~b}$ above.

(1.4) And then comes Terwilliger algebras... In 1992 Paul Terwilliger published the first of a series of three papers on the subconstituent algebra of a CC. In the case of commutative ASs, this algebra extends the adjacency algebra of the AS by a copy of its dual algebra.

Definition 3. [47] Let $S=\left\{A_{0}=I, A_{1}, \ldots, A_{r-1}\right\}$ be set of adjacency matrices for a $C C$ (or $A S$ ) of rank $r$ and order $n$.

Fix a vertex $x$ in the $A S S$ (i.e. the index of a designated row). For each $A_{i}$, let $E_{i}^{*}=E_{i}^{*}(x)$ be the diagonal idempotent matrix whose yy-entry is 1 if $\left(A_{i}\right)_{x y}=1$, and otherwise 0 . If $E^{*}(x)=\left\{E_{i}^{*}(x): i=0,1, \ldots, r-1\right\}$ is this dual idempotent basis then $\mathbb{Q} E^{*}(x)$ is an $r$-dimensional semisimple commutative $\mathbb{Q}$-algebra.

The rational Terwilliger (or subconstituent) algebra at vertex $x$ is the $\mathbb{Q}$-subalgebra of $M_{n}(\mathbb{Q})$ generated by the set of 01-matrices $S \cup E^{*}(x)$.

At this point we invite the reader to compute that for $S=K_{n}$ and each choice of $x, T_{x}(S)$ is a 5 -dimensional coherent algebra. In general, there is one $T_{x}(S)$ up to isomorphism for each orbit of the combinatorial automorphism group $A u t(S)$. When $x$ and $y$ are not in the same orbit of $\operatorname{Aut}(S)$, it may be the case that $T_{x}(S)$ and $T_{y}(S)$ are not isomorphic, and can even have different dimension. $T_{x}$ is a semisimple subalgebra of a coherent algebra (its coherent closure), but it is often the case that it is not coherent.

Terwilliger algebras have been an area of intense study for SRGs and DRGs since they were first introduced (see the survey [43]). Deep connections to orthogonal polynomials were soon realized as generators of the Terwilliger algebras were found to satisfy the same identities as the universal algebras of the quantum groups related to certain families of orthogonal polynomials. This led to a characterization of irreducible representations in many of these casee, and to interesting 
connections of ASs to the spin models of conformal field theory (see Bannai's recent survey [5].) The solution of a rationality problem for Terwilliger algebras was a key step in the recent solution of the Bannai-Ito conjecture by Bang, Dubickas, Koolen, and Moulton showing that there are a finite number of association schemes of rank $>3$ that are both metric and cometric (i.e. the dual is also metric) [3].

The rational Terwilliger algebra for a thin AS (i.e. a finite group $G$ ) of order $n$ will be isomorphic to the full matrix algebra $M_{n}(\mathbb{Q})$. For any other AS, the dimension of the Terwilliger algebra $T_{x}(S)$ exceeds the rank of $S$ for every $x$. For SRGs (symmetric ASs of rank 3), Yamazaki and Tomiyama proved

Theorem 4. [50] For a symmetric rank $3 A S$ (i.e. $b_{1}$ is a $S R G$ ), and any vertex $x$,

$$
\mathbb{C} \otimes T_{x}(S) \simeq M_{3}(\mathbb{C}) \oplus M_{2}(\mathbb{C})^{m_{2}(x)} \oplus \mathbb{C}^{m_{1}(x)},
$$

where $m_{1}(x)$ and $m_{2}(x)$ can be determined from certain eigenspaces of a principal submatrix of $b_{1}$.

The Terwilliger algebra of an AS of rank $r$ always has one simple component isomorphic to $M_{r}(\mathbb{Q})$. This is the component corresponding to the principal irreducible $T_{x}(S)$-module generated by the elementary basis vector $e_{x}$ [47]. It is easy to see that this module restricts to the regular module for both $\mathbb{Q} S$ and the $r$-dimensional diagonal commutative algebra $\mathbb{Q}\left[E^{*}(x)\right]$. The nonprincipal irreducible $T_{x}(S)$-modules are much more mysterious. Though it is rare, these can have dimension equal or exceeding the rank of $S$.

On the other hand, very little work has been done on Terwilliger algebras for general ASs and CCs, other than the very recent work of Muzychuk and Xu giving a formula for the Terwilliger algebra $T_{x}(S w r U)$ of the wreath product of two CCs $S$ and $U$ in terms of $T_{x}(S)$ and $T_{x}(U)$. For the Terwilliger algebras of DRTs, the author has recently asked:

Question 3. Suppose $S$ is an asymmetric $A S$ of rank 3 and order $4 u+3, u \geq 0$, (i.e. a DRT), and $x$ is a vertex of $S$.

- Is $\operatorname{dim} T_{x}(S)$ always odd and $\leq 8 u+9$ ?

- Is the principal irreducible $T_{x}(S)$-module the unique one of maximal dimension 3 ?

Much of the interest in Terwilliger algebras stems from the fact that they are much more powerful than the spectrum in distinguishing cospectral (directed) graphs up to isomorphism. The author has found families of cospectral DRTs of order 27 that are not distinguished by their complex Terwilliger algebras, but are distinguished by their rational Terwilliger algebras. 
Question 4. Except for finite groups, are CCs distinguished combinatorially by their rational Terwilliger algebras (as a list of algebras up to isomorphism)?

The study of integral or modular Terwilliger algebras is completely open. In fact there is still only one mention of integral Terwilliger algebras in the literature, in one of Terwilliger's conjectures:

Question 5. [49, Conjecture 10, first part] Is $\mathbb{Z}\left\langle S \cup E^{*}\right\rangle$ equal to the set of all integral matrices in $T_{x}(S)$ ?

\section{Their Representation Theory}

(2.1) Integrality. Let $\mathbf{B}=\left\{b_{0}, b_{1}, \ldots, b_{r-1}\right\}$ be the set of adjacency matrices of a CC, AS, or the standard basis of a SITA. Then $\mathbf{B}^{2} \subset \mathbb{N} \mathbf{B}$ implies $\mathbb{Z} \mathbf{B}$ is a ring, and so $R \mathbf{B}:=R \otimes_{\mathbb{Z}} \mathbb{Z} \mathbf{B}$ is an $R$-algebra for any commutative ring $R$. As we do for group algebras, this perspective allows us to consider representation theory over any ring or field, like $\mathbb{C}, \mathbb{R}, \mathbb{Q}, \mathbb{Z}$, finite fields $\mathbb{F}_{p^{n}}$, or $p$-adic fields $\hat{\mathbb{Q}}_{p}$.

An analogue of Maschke's theorem gives a nice semisimplicity condition. Let $\Psi$ be the sum of the inequivalent irreducible representations of $\mathbb{C B}$. The Frame number of a SITA basis $\mathbf{B}$ is the absolute value of its discriminant in the representation $\Psi$; i.e. $\mathcal{F}(\mathbf{B})=\left|\operatorname{det}\left(\left(\operatorname{trace}\left(\Psi\left(b_{i} b_{j}\right)\right)\right)_{i, j}\right)\right|$. We can readily see this is a positive integer. In terms of the order $n$, rank $r$, degrees $\delta\left(b_{i}\right)$ for $i=0,1, \ldots, r-1$, multiplicities $m_{\chi_{j}}$ and character degrees $\chi_{j}(1)$ for $\operatorname{Irr}\left(\mathbb{C B}=\left\{\delta=\chi_{0}, \chi_{1}, \ldots, \chi_{k}\right\}\right.$, its formula is

$$
\mathcal{F}(\mathbf{B})=\frac{n^{r}\left(\delta\left(b_{1}\right) \cdots \delta\left(b_{r-1}\right)\right)}{\left(m_{c h i_{1}}^{\chi_{1}(1)} \cdots m_{\chi_{k}}^{\chi_{k}(1)}\right)} .
$$

Theorem 5. ([2], see also [23]) Let $\mathbf{B}$ be the standard basis of a SITA, and let $F$ be a field. Then $F \mathbf{B}$ is semisimple $F$-algebra iff the characteristic of $F$ is 0 or does not divide $\mathcal{F}(\mathbf{B})$.

Terwilliger algebras are semisimple in characteristic zero. To date there has been no treatment of Terwilliger algebras in prime characteristic.

(2.2) Fields of character values. Being finite-dimensional and semisimple, the inequivalent irreducible representations of SITAs over $\mathbb{C}$ are determined by the irreducible characters. In 1978, Norton posed the following question, originally for commutative ASs, which has since been extended to all ASs (which would imply it for CCs): 
Question 6. [Cyclotomic Eigenvalue Conjecture (CEC)] Suppose $\mathbf{B}$ is the standard basis of a CC. Let $\chi \in \operatorname{Irr}(\mathbb{C B})$. Then $\forall b_{i} \in \mathbf{B}$, is $\chi\left(b_{i}\right)$ an element of a cyclotomic extension of $\mathbb{Q}$ ? (The conjecture would say the answer is yes.)

It is easy to see that the conclusion of CEC holds for finite groups and all ASs of rank 2 or 3. But it does not hold for regular graphs of order $\geq 9$, nor SITAs of order $\geq 13$. It could even fail for ASs of prime order. In [39] Komatsu gave examples of number fields that make it possible for the conditions for noncyclotomic ASs of prime order to exist discovered by Hanaki and Uno in [28] to be satisfied. Rational Terwilliger algebras of ASs of rank $\geq 3$ need not have cyclotomic fields of character values. On the other hand, the conclusion of the CEC does hold for two-orbit CCs, and for the fusion rings occurring in integral modular data [17].

(2.3) Rational representation theory. If $\mathbf{B}$ is the standard basis of an AS, CC, or SITA, then $\mathbb{Q}$ B is semisimple, so we can ask for information such as the simple components of its Wedderburn decomposition, such as the rational Schur indices $m(\chi)$ of its irreducible characters. Another natural question is to classify the algebras that can occur as a simple component of $\mathbb{Q B}$ for some AS, CC, or SITA. For finite groups this is answered (for the most part) by the collections of Schur subgroups of the Brauer groups of cyclotomic number fields. For ASs resolution of the CEC is a fundamental first step toward a solution to this problem. For ASs and SITAs Rahnamai-Barghi and the author initiated a study of Schur indices in [33] that established several basic results, as well as the effectiveness of the Clifford theory reduction for computing Schur indices of ASs.

Theorem 6. [33] (i) If $\chi \in \operatorname{Irr}(\mathbb{C B})$ and $\mathbb{Q}(\chi) \subset \mathbb{R}$, then $m(\chi) \leq 2$.

(ii) The collection of Brauer equivalence classes of $K$-central simple algebras that occur as simple components of $\mathbb{Q} \mathbf{B}$ for some $A S$ (or $T A$ ) $\mathbf{B}$ form a subgroup of the Brauer group of K, which we call the AS-(TA-)Schur Subgroup of Br $(K)$.

For finite groups, it is a fact that $m(\chi)^{2}$ always divides $|G|$. Despite calculating Schur indices for many ASs in Hanaki and Miyamoto's classification, we have yet to be able to formulate any statement of this nature.

Question 7. Is there a bound for $m(\chi)$ in terms of parameters of $\mathbf{B}$ ?

(2.4) Modular representation theory. Let $\mathbf{B}$ be a SITA, and suppose $F$ is a field of characteristic $p$ dividing the Frame number of B. By the Krull-SchmidtAzumaya Theorem for algebras over fields (see $[18,(6.12)]$ ), the indecomposable summands (blocks) of $F \mathbf{B}$ are well-defined. By the Jordan-Hölder theorem ([18, (3.11)]), the irreducible $F \mathbf{B}$-modules within each block are well-defined up to isomorphism. Along the same line of approach to block theory for modular group 
algebras, Hanaki has initiated the use of $p$-modular systems $(K, R, F)$ to study modular representations of adjacency algebras of ASs in characteristic $p>0$. $(K, R, F)$ is a splitting $p$-modular system for an AS $\mathbf{B}$ when $K$ is a finite extension of the $p$-adic completion of the rationals that splits $\mathbb{Q B}, R$ is its ring of integers, and $F$ the residue field of $R$. In this situation one has the idempotent lifting property from $F \mathbf{B}$ to $R \mathbf{B}$, and so as with groups one can define the blocks either in terms of indecomposable summands of $F \mathbf{B}$ or $R \mathbf{B}$, or with a partition of simple components of $K \mathbf{B}$. Some control of the situation is sacrificed in generalizing to ASs (or SITAs), in particular $F \mathbf{B}$ is not necessarily a symmetric algebra, but its Cartan matrix is still symmetric (so decomposition numbers are well-defined), and the blocks of $F \mathbf{B}$ can depend on $p$ and the choice of $p$-modular system (not just on $p$ as happens for the group algebras of finite groups). Hanaki has posed the following problems in the context of ASs, which we (perhaps naïvely) generalize here to the setting of SITAs as the fundamentals of modular representation theory of SITAs have yet to appear in the literature!

Question 8. [21] Let $\mathbf{B}$ be an AS (or standard basis of a SITA), and let (K, R,F) be a splitting p-modular system for $\mathbf{B}$.

(i) Which F-algebras can occur as blocks of $F \mathbf{B}$ ?

(ii) When does FB have finite representation type?

(iii) Which facts about Cartan matrices of modular group algebras generalize to adjacency algebras of ASs (or SITAs)?

(iv) Is it possible to develop a defect theory for blocks of adjacency algebras of ASs (or SITAs)?

Note that (i) is known for modular group algebras of finite groups, as there are several major conjectures of modular representation theory that deal with aspects of this problem - for an up-to-date account of the status of the main conjectures of block theory, see [40].

The best results established up to now in modular representation theory for adjacency algebras of finite association to date concern the indecomposable summands of the standard module of the adjacency algebra of an association scheme in characteristic $p$. The most significant of these is the following "locality theorem":

Theorem 7. [22], [9] Let $\mathbf{B}$ be the standard basis of a SITA with positive rational multiplicites (such as the set of adjacency matrices of an association scheme). Suppose the order of $\mathbf{B}$ is a power of the prime $p$, and $F$ is a field of characteristic $p$. Then $F \mathbf{B}$ is a local ring.

This locality theorem was a key step in the classification of association schemes of order $p$ (see [28]). 
Prior to Hanaki's work, the concept of $p$-rank was used to distinguish cospectral DRGs in some cases (see [14]). Sometimes an AS B can have elements $b_{i}$ for which the standard matrix of $b_{i}$ has smaller matrix rank modulo a prime $p$ than it does in characteristic zero. But to date no connection has been made between $p$-ranks of elements of $\mathbf{B}$ and representations of $\mathbf{B}$ over fields of characteristic $p$.

(2.5) Units of $\mathbb{Z B}$. When $\mathbf{B}$ is an AS or the standard basis of a SITA, we can ask about the structure of the unit group $U(\mathbb{Z} \mathbf{B})$. For finite groups $G$, this group is known to be finitely generated, and much work has been done on the problem of how to find generators for a subgroup of finite index. For SITAs, one can show the basis elements of degree 1 form a group $L(\mathbf{B})$, but it is rare for any of the other basis elements to even be invertible matrices in the standard representation, let alone units of $\mathbb{Z} \mathbf{B}$. Though it is expected $U(\mathbb{Z} \mathbf{B})$ is finitely generated, it is likely to be a relatively smaller group than what we see for unit groups of group algebras of the same order.

Gurmail Singh and the authour have established the analogue of one of the most basic results about units of integral group rings:

Theorem 8. [34] Let $\mathbf{B}$ be the standard basis of a SITA. Then the central torsion units of $\mathbb{Z} \mathbf{B}$ are trivial: (i.e. $u= \pm b_{i}$ for some $b_{i} \in L(\mathbf{B})$ ).

There are many fundamental properties of units of integral group rings that are yet to be established for integral adjacency rings of ASs or SITAs. For commutative SITAs, a natural question is to ask for a generalization of the formula for the torsion-free rank of the unit group of the integral group ring.

Question 9. If $\mathbf{B}$ is a commutative SITA, is there a formula for the rank of the torsion-free part of $U(\mathbb{Z} \mathbf{B})$ in terms of parameters of $\mathbf{B}$ ?

As with integral group rings, one can study the normalized units of $\mathbb{Z} \mathbf{B}$, i.e. the units having degree 1. One can ask for the generalizations of the Zassenhaus conjectures for finite subgroups of these units. However, the main tool available for studying normalized torsion units in integral group rings, the Luthar-Passi method, is not available because it is not clear what to use for conjugacy classes of SITAs unless the SITA has a central fusion (see [52]).

Since the standard basis elements of degree 1 in a SITA $\mathbf{B}$ form a group $L(\mathbf{B})$, and standard basis elements of degree $>1$ do not lie in $U(\mathbb{Z B})$, we can also ask:

Question 10. Is there a relationship between $U(\mathbb{Z} \mathbf{B})$ and $U(\mathbf{Z}(L(\mathbf{B}))$ ? If $L(\mathbf{B}) \neq$ $\mathbf{B}$, can $U(\mathbb{Z} L(\mathbf{B}))=U(\mathbb{Z} \mathbf{B})$ ?

(2.6) The Zeta function of the $\mathbb{Z}$-order $\mathbb{Z} \mathbf{B}$. When $\mathbf{B}$ is a SITA, $\mathbb{Q} \mathbf{B}$ is a semisimple algebra, so $\mathbb{Z} \mathbf{B}$ is a $\mathbb{Z}$-order. The order-theoretic situation for $\mathbb{Z} \mathbf{B}$ 
is similar to what happens for integral group rings of finite groups. Since there is a character-theoretic formula for the centrally primitive idempotents $e_{\chi}, \chi \in$ $\operatorname{Irr}(\mathbb{C B})$ of $\mathbb{C B}[9]$, one can determine a maximal order containing $R \mathbf{B}$ for a suitable ring of algebraic integers $R$.

Recall that the zeta function of a $\mathbb{Z}$-module $M$ is the function $\zeta_{M}(s)=\sum_{n} a_{n} s^{-n}$, where $a_{n}$ is the number of $\mathbb{Z}$-submodules of $M$ with index $n$ for all $n \geq 1$. In particular this applies to the $\mathbb{Z}$-order $\mathbb{Z} \mathbf{B}$. For integral group rings of finite groups, this line of research was initiated by Solomon [42] and extended by work of Reiner [45], Hironaka [37], and Takegahara [46] between 1977 and 1987. Hanaki and Hirasaka generalized the idea to small ASs in 2015.

Theorem 9. The zeta functions of the order $\mathbb{Z} \mathbf{B}$ have been computed for the following ASs:

- [42] $\mathbf{B}=C_{p}$, a cyclic group of prime order.

- $[45] \mathbf{B}=C_{p^{2}}$.

- [37] $\mathbf{B}=C_{p} \rtimes C_{q}, p \neq q$ distinct primes.

- $[46] \mathbf{B}=C_{p} \times C_{p}$.

- $[24] \mathbf{B}=K_{n}$.

- [24] $o(\mathbf{B})=p$ a prime.

- [30] $\mathbf{B}=\mathbf{C} \times \mathbf{D}$, where the orders $\mathbb{Z} \mathbf{C}$ and $\mathbb{Z} \mathbf{D}$ are "locally coprime".

In these results, the formulas for $\zeta_{\mathbb{Z} \mathbb{B}}(s)$ are expressed as multiples of powers of zeta functions of the ring of integers of certain number fields. For example, Solomon's formula for the zeta function of the integral group ring $\mathbb{Z} C_{p}$ for a cyclic group of prime order $p$ is

$$
\zeta_{\mathbb{Z} C_{p}}(s)=\left(1-p^{-s}+p^{1-2 s}\right) \zeta_{\mathbb{Z}}(s) \zeta_{\mathbb{Z}\left[\omega_{p}\right]}(s),
$$

where $\omega_{p}$ is a primitive complex $p$-th root of unity and $\mathbb{Z}\left[\omega_{p}\right]$ is the ring of integers of the splitting field of a non-principal irreducible character of $C_{p}$. Hanaki and Hirasaka's formula for the zeta function of $\mathbb{Z} S$, where $S$ is an association scheme of order $p$, is very similar. Their formula for the zeta function of $\mathbb{Z} K_{n}$ where $n$ has prime factorization $p_{1}^{m_{1}} p_{2}^{m_{2}} \cdots p_{k}^{m_{k}}$ is

$$
\zeta_{\mathbb{Z} K_{n}}(s)=\zeta_{\mathbb{Z}}(s)^{2} \prod_{i=1}^{k}\left(p_{i}^{m_{i}} t^{2 m_{i}}+\sum_{j=0}^{m_{i}-1} p_{i}^{j} t^{2 j}(1-t)\right) .
$$

Question 11. Can we compute the zeta function of $\mathbb{Z} \mathbf{B}$, where $B$ is an $A S$ of rank 3 (i.e. an $S R G$ or DRT)?

Remark 1. At the GARC program at ICTS Bangalore in October 2019, Uri Onn asked if the zeta function in any of the above cases satisfies a functional equation, 
such as the one $\zeta(s)=\zeta(1-s)$ satisfied for the zeta function of $\mathbb{Z}$ or the ones satisfied by the groups of elliptic curves over algebraicially closed fields of prime characteristic. Functional equations for general $\mathbb{Z}$-orders, which leads to their analytic continuation to the plane, were conjectured by Solomon were established by Bushnell and Reiner [16]. This aspect has yet to be explored in the special case of integral adjacency rings of ASs or SITAs.

\section{Their Algebraic Structure}

(3.1) Closed subsets and primitivity. Suppose $\mathbf{B}$ is an AS or the standard basis of a TA. A sub-AS or sub-TA is a closed subset $\mathbf{D}$ of $\mathbf{B}$, satisfying $\mathbf{D}^{*}=\mathbf{D}$, $\mathbf{D}^{2} \subset \mathbb{N D}$, and $\operatorname{dim} \mathbb{C D}=|\mathbf{D}|$. Every closed subset of a SITA is a SITA, and a closed subset of an AS is an AS [55]. When $\mathbf{D}$ is a closed subset, we write $\mathbf{D}^{+}$for the sum of the elements of $\mathbf{D}$. Every $b \in \mathbf{B}$ determines a (left) coset $b \mathbf{D}=\left\{c \in \mathbf{B}: \lambda_{b d c}>0\right.$ for some $\left.d \in \mathbf{D}\right\}$, and similarly we define right cosets and double cosets. The order of the closed subset $\mathbf{D}$ is $o(\mathbf{D})=\delta\left(\mathbf{D}^{+}\right)=\sum_{d \in \mathbf{D}} \delta(d)$. The next theorem, which generalizes Lagrange's Theorem for finite groups, is very useful.

Theorem 10 ([55], [9]). Suppose $\mathbf{D}$ is a closed subset of an AS (or TA) B.

(i) $\mathbf{B}$ is partitioned into left $\mathbf{D}$-cosets $b \mathbf{D}, b \in \mathbf{B}$ (using what is analogous to support in group rings).

(ii) $\mathbf{B}$ is partitioned into $\mathbf{D}-\mathbf{D}$-double cosets $(\mathbf{D} b \mathbf{D})$.

A TA $\mathbf{B}$ is primitive if it contains no closed subsets other than $\left\{b_{0}=I\right\}$ and itself. The only primitive finite groups are the $C_{p}$ 's where $p$ is prime.

Theorem 11. (a) [28] Any AS or SITA of prime order $p$ will be primitive (and commutative and of rank dividing $p-1$ ).

(b) [32] Every noncommutative TA of rank 5 is primitive (but such a TA cannot be a SITA).

(c) [31] There are primitive noncommutative SITAs of rank 6 .

Examples of primitive noncommutative ASs can be constructed using noncommutative double coset algebras corresponding to maximal subgroups of finite groups. Such examples are quite rare, in fact there is only one such subgroup for a group of order $<1000$, which occurs for $G=P S L(2,11)$, it has rank 9 and order 55. The smallest rank known to occur for a primitive noncommutative SITA is 7 , it has order 560 and was discovered by Jason Williford. 
Question 12. (Muzychuk) Can a primitive noncommutative AS have rank 6 ?

(3.2) Quotients and homomorphisms. Unlike the case for groups, quotients of ASs and TAs can result from any closed subset.

Theorem 12 ([55], [2]). Let $\mathbf{B}$ be an $A S$ or the standard basis of a TA. Let $\mathbf{D}$ be any closed subset of $\mathbf{B}$. Then $\mathbf{B} / / \mathbf{D}=\left\{o(\mathbf{D})^{-1}(\mathbf{D} b \mathbf{D})^{+}: b \in \mathbf{B}\right\}$ is the set of standard matrices of an $A S$ (or TA) of order $o(\mathbf{B}) / o(\mathbf{D})$.

For ASs, it follows that $o(\mathbf{D})$ divides $o(\mathbf{B})$, but in the case of standard bases of SITAs, the order of a quotient need not be an integer (see [10]).

Zieschang gave the first somewhat technical definitions of scheme morphism in [55] and scheme homomorphism in [54]. The latter was used by Hanaki to formulate a category of association schemes with base point in [20]. Blau introduced the notion of TA-homomorphism in [9], which extends both Zieschang's notion of AShomomorphisms and is compatible with Arad-Fisman-Muzychuk's TA-quotients.

Definition 13. An $A S$ (or TA) homomorphism $\phi: \mathbf{B} \rightarrow \mathbf{C}$ is determined by a composition which (up to positive scalars) takes

$$
\mathbf{B} \rightarrow \mathbf{B} / / \mathbf{D} \rightarrow \mathbf{E} \hookrightarrow \mathbf{C},
$$

for some closed subset $\mathbf{D}$ of $\mathbf{B}\left(\mathbf{D}=\operatorname{ker} \phi:=\left\{b \in \mathbf{B}: \phi(b)=\delta(b) \phi\left(b_{0}\right)\right\}\right)$ and some closed subset $\mathbf{E}$ of $\mathbf{C}$.

A second notion of AS-homomorphism was studied by French in [19] that also gives a viable category of association schemes with base point which extends the finite group category. By restricting his AS-homomorphisms to only those whose kernels are normal closed subsets, French obtained a category of association schemes with base point that has the additional property that every AS-homomorphism $\mathbf{B} \rightarrow \mathbf{C}$ always induces an algebra homomorphism $\mathbb{C B} \rightarrow \mathbb{C}$.

Algebraically, an AS- or TA-homomorphism as defined above does not produce an algebra homomorphism from $R \mathbf{B}$ to $R[\mathbf{B} / / \mathbf{D}]$ unless $\mathbf{D}$ is a normal closed subset, i.e. $b \mathbf{D}=\mathbf{D} b$ for all $b \in \mathbf{B}$. When $F$ is a field of characteristic zero, a AS- or TAhomomorphism $F[\mathbf{B} / / \mathbf{D}]$ is a semisimple algebra whose simple components are of smaller dimension and are Morita equivalent to certain simple components of $F \mathbf{B}$. Over $\mathbf{C}$, a result of Hanaki and Hirasaka shows $\mathbb{C}[\mathbf{B} / / \mathbf{D}] \simeq e_{\mathbf{D}} \mathbb{C B} e_{\mathbf{D}}$, where $e_{\mathbf{D}}$ is the centrally primitive idempotent of $\mathbb{C D}$ corresponding to the degree map of $\mathbf{D}$ [25]. An AS-homomorphic image of a finite group $G$ will produce a double coset algebra $\mathbb{C}[G / / H]$ when the kernel is a subgroup $H$. These are a special case of the two-orbit CCs, and known as Schurian ASs. 
Question 13. Let $\mathbf{D}$ be a closed subset of an $A S \mathbf{B}$.

(i) Is there a relationship between the unit groups $U(\mathbb{Z} \mathbf{B})$ and $U(\mathbb{Z}[\mathbf{B} / / \mathbf{D}]$ ? (The answer to this question is is not known for finite groups.)

(ii) Are there a relationships between the zeta functions $\zeta_{\mathbb{Z} \mathbf{D}}(s), \zeta_{\mathbb{Z} \mathbf{B}}(s)$, and $\zeta_{\mathbb{Z}[\mathbf{B} / / \mathbf{D}]}(s)$ ?

(3.3) Extensions. Suppose $\mathbf{B}=\left\{b_{0}, b_{1}, \ldots, b_{r-1}\right\}$ and $\mathbf{C}=\left\{c_{0}, c_{1}, \ldots, c_{s-1}\right\}$ are two ASs (or standard bases of TAs). The following constructions give new ASs (or TAs), with the indicated standard bases:

- (direct product) $\mathbf{B} \times \mathbf{C}=\left\{b_{i} \otimes c_{j}: i=0, \ldots, r-1 ; j=0, \ldots, s-1\right\}$.

- (wreath product) $\mathbf{B} \imath \mathbf{C}=\left\{b_{i} \otimes c_{0}(i=0, \ldots, r-1), \mathbf{B}^{+} \otimes c_{j}:(j=1, \ldots, s-1)\right\}$.

- (semidirect product) $\mathbf{B} \rtimes G=\left\{b_{i} g: i=0, \ldots, r-1 ; g \in G\right\}$ for a group homomorphism $G \rightarrow \operatorname{Aut}(\mathbf{B})$, with multiplication given by the linearization of the identity $\left(b_{i} g\right)\left(b_{j} h\right)=\left(b_{i} b_{j}^{g}\right)(g h)$.

- $(\psi$-generalized wreath product $) \mathbf{B} \imath_{\psi} \mathbf{C}$ for a TA homomorphism $\psi: \mathbf{B} \rightarrow \mathbf{C}$. The table algebra basis is (up to standard renormalization) $\left(\operatorname{ker}_{\mathbf{B}}(\psi)-\left\{b_{0}\right\}\right) \cup \mathbf{C}=$ $\left(\left\{b_{i}: \psi\left(b_{i}\right) \in \mathbb{R}^{+} c_{0}\right\}-\left\{b_{0}\right\}\right) \cup\left\{\delta\left(\psi\left(b_{i}\right) c_{j_{i}}:\left\{c_{j_{i}}\right\}=\operatorname{Supp}\left(\psi\left(b_{i}\right)\right), i=0, \ldots, r-1\right\} \cup\right.$ $\left\{c_{j}: c_{j} \notin \operatorname{Supp}(\psi(\mathbf{B})\}\right.$. The products of pairs of elements in $\mathbf{B}$ or $\mathbf{C}$ are as in these ASs, and for $b_{i} \in \operatorname{ker}_{\mathbf{B}}(\psi)$ and $c_{j} \in \mathbf{C}-\operatorname{Supp}(\psi(\mathbf{B})), b_{i} c_{j}=\delta\left(b_{i}\right) c_{j}=c_{j} b_{i}$. The special case where $\psi\left(b_{i}\right)=\delta\left(b_{i}\right) c_{0}$ for $i=0, \ldots, r-1$ produces the wreath product. This construction has appeared under various names in the literature. It was first introduced in [1], reintroduced under the name circle product by Blau and Chen in [11], and an new version of it was recently studied by [51] under the name wedge-direct product.

Though the $\psi$-generalized wreath product construction model produces many extensions, it still falls short of being a replacement for group cohomology in explaining group extensions for the AS or TA setting. An interesting attempt to explain extensions of ASs by generalizing group cohomology was produced by Bang and Hirasaka in [4], it produced some interesting extensions of ASs that result from combinatorial difference sets that have yet to be explained by the other constructions. The problem of finding a general theory for extensions of ASs and TAs continues to attract attention of algebraists working in this area.

(3.4) Fusions. A fusion of a TA $\mathbf{B}$ is a TA $\mathbf{D}$ obtained from the characteristic functions of a $*$-compatible partition of $\mathbf{B}$, with one of the subsets of the partition being $\left\{b_{0}\right\}$. For fusions of a CC, we need to combine a partition of $\Delta$ with a *-compatible partition of $\mathbf{B}-\Delta$ to produce the basis of a minimal dimensional subalgebra. 
The fusions of thin ASs (group rings) are precisely what are known as unital Schur rings. The most well-known example of a fusion of an AS is the center of the group algebra of a finite nonabelian group, which is the fusion created from the partition of the finite group $G$ into its conjugacy classes. Other well-known fusions of the group basis $G$ of a group algebra arise from partitions constructed from $H$-orbits where $H \leq A u t(G)$, or from the sets $N_{i}-N_{i-1}$ arising from an ascending chain $1=N_{0} \leq N_{1} \leq N_{2} \leq \cdots \leq N_{k}=G$ of normal subgroups of $G$.

Remark 2. The "*-compatibility condition" on partitions that produce a fusion cannot be dropped. For example, in the group algebra $\mathbb{C} S_{3}$, the set

$$
\mathbf{D}=\{(1),(1,2,3)+(1,2),(1,3,2)+(1,3),(2,3)\}
$$

generates a 4-dimensional subalgebra $\mathbb{C D}$ of $\mathbb{C} S_{3}$, which is not semisimple! This is an example of what we call a semifusion.

Calculation of the complete fusion lattice of an AS or SITA of rank larger than 12 is complicated by the difficult computational problem of searching the set of partitions of a set of size 13 or more. Even among the ASs of order up to 30 in the classification, the entire fusion lattice for orders 24,28 , and 30 is yet to be completely determined.

The opposite process to fusion is fission. $\mathbf{D}$ is a fission of $\mathbf{B}$ iff $\mathbf{B}$ is a fission of D. Being able to predict character values of fusions and fissions of ASs has been an important theme in attempts to resolve the CEC conjecture (see [8]).

Question 14. If $\mathbf{D}$ is a fusion (or semifusion) of an $A S$ (or SITA) $\mathbf{B}$, is there a relationship between $U(\mathbb{Z} \mathbf{D})$ and $U(\mathbb{Z} \mathbf{B})$ ? If $\mathbf{B}$ is commutative, can we find a relationship between the torsion-free ranks of these groups?

(3.5) The Three Types of ASs. ASs can be divided into three types:

(Type 1) the Schurian ASs are the AS homomorphic images of finite groups;

(Type 2) the fusions of Schurian ASs are the unital and non-unital Schur rings, and

(Type 3) the ASs with intransitive automorphism groups are homogeneous fusions of CCs with no intermediate AS fusion that are not included in (i) and (ii).

The (combinatorial) automorphism group of an CC of order $n$ is the subgroup of permutation matrices that centralize every standard basis matrix of the CC. These permutation matrices act on the set of $n$ vertices of the AS, and we say the $\mathrm{CC}$ has a transitive automorphism group when this action is transitive. ASs of types 1 and 2 have transitive automorphism groups, and indeed in these cases 
the permutation matrices required for Proposition 2 can be chosen to be those of an order $n$ group of permutations consisting of the identity matrix and $n-1$ derangements. The standard basis matrices of ASs of type 3 do not sit inside the span of any collection of permutation matrices forming a group of order $n$. Their automorphism groups have $k>1$ orbits on vertices, and the AS is a homogeneous fusion of a CC with $k$ fibers. Unlike the case for small ASs, it is the case that most ASs have intransitive automorphism groups! In the worst case scenario, an AS scheme of type 3 can be even be a homogeneous fusion of the full matrix CC (with $n$ fibers) having no intermediate fusion between it and the full matrix CC. In the opinion of this authour, it is hard to conceive of any possible restriction on the character values of ASs of type 3 that are direct fusions of the full matrix CC. However, we have yet to find any ASs with intransitive automorphism groups that violate the CEC.

Remark 3. The CEC holds for ASs in family (i) but is open for (ii) and (iii). That the CEC holds for fusions of commutative ASs with cyclotomic fields of character values is a consequence of the Bannai-Muzychuk character table criterion [6], [44].

Question 15. (i) Does the CEC hold for Schur rings that are fusions of nonAbelian finite groups?

(ii) Can an element of a semifusion of a group ring basis have noncyclotomic eigenvalues?

\section{References}

[1] Z. Arad and E. Fisman, On table algebras, C-algebras, and applications to finite group theory, Comm. Algebra, 19, (1991), 2955-3009.

[2] Z. Arad, E. Fisman, and M. Muzychuk, Generalized table algebras, Israel J. Math., 114 (1999), 29-60.

[3] S. Bang, A. Dubickas, J. Koolen, and V. Moulton, There are only finitely many distance-regular graphs of fixed valency greater than two, Adv. Math., 269 (2015), 1-55.

[4] S. Bang and M. Hirasaka, Construction of association schemes from difference sets, European J. Combin., 26 (1), (2005), 59-74.

[5] E. Bannai, Combinatorics as Pure Mathematics - Aims of Algebraic Combinatorics, Sugaku, 62 (4), (2010), 433-452. (Japanese, English version at http://math.sjtu.edu.cn/conference/Bannai/2012/data/journal.pdf) 
[6] E. Bannai, Subschemes of some association schemes, J. Algebra, 144 (1), (1991), 167-188.

[7] E. Bannai and T. Ito, Algebraic Combinatorics I: Association Schemes, Benjamin Cummings, Menlo Park, CA, 1984.

[8] E. Bannai and S.-Y. Song, Character tables of fission schemes and fusion schemes, European J. Combin., 14 (5), (1993), 385-396.

[9] H. Blau, Table algebras, European J. Combin., 30, (2009), 1426-1455.

[10] H. Blau, Quotient structures in C-algebras, J. Algebra, 175 (1995), 24-64; Erratum: 177 (1995), 297-337.

[11] H. Blau and G. Chen, Reality-based algebras, generalized Camina-Frobenius pairs, and the non-existence of degree maps, 40 (4), (2012), 1547-1562.

[12] A. Brouwer, BCN tables, https://www.win.tue.nl/ aeb/drg/drgtables.html

[13] A. Brouwer, A. Cohen, and A. Neumaier, Distance Regular Graphs, SpringerVerlag, Berlin, 1989.

[14] A. Brouwer and C.A. van Eijl, On the p-rank of the adjacency matrices of strongly regular graphs. J. Algebraic Combin., 1 (4), (1992), 329-346.

[15] G. Birkhoff, Three observations on linear algebra. Univ. Nac. Tucumán. Revista A, 5 (1946). 147-151. (Spanish)

[16] C. Bushnell and I. Reiner, Zeta functions of arithmetic orders and Solomon's conjectures, Math. Z., 173, (1980), 135-161.

[17] A. Coste and T. Gannon, Remarks on Galois in rational conformal field theories, Phys. Lett. B, 323, (1994), 316-321.

[18] C. Curtis and I. Reiner, Methods of Representation Theory: With applications to groups and orders, Vol. I, Wiley-Interscience, New York, 1991.

[19] C. French, Functors from association schemes, J. Combin. Theory Ser. A, 120 (6), (2013), 1141-1165.

[20] A. Hanaki, A category of association schemes, J. Combin. Theory Ser. A, 117 (8), (2010), 1207-1217.

[21] A. Hanaki, Representation theory of finite association schemes, European J. Combin., 30 (6), (2009), 1477-1496. 
[22] A. Hanaki, Locality of a modular adjacency algebra of an association scheme of prime power order, Arch. Math. (Basel), 79 (3), (2002), 167-170.

[23] A. Hanaki, Semisimplicity of adjacency algebras of association schemes, $J$. Algebra, 225, (2000), 124-129.

[24] A. Hanaki and M. Hirasaka, The zeta function of the integral adjacency algebra of association scheme of prime order or rank 2, Hokkaido J. Math., 46 (1), (2015), 75-91.

[25] A. Hanaki and M. Hirasaka, Theory of Hecke algebras to association schemes, SUT J. Math., 38 (1), (2002), 61-66.

[26] A. Hanaki, H. Kharaghani, A. Mohammadian, and B. TayfehRezaie, Classification of skew-Hadamard matrices of order 32 and association schemes of order 31, preprint, available at http://math.ipm.ac.ir/t̃ayfeh-r/papersandpreprints/SH32AS31r.pdf

[27] A. Hanaki and I. Miyamoto, Classification of Small Association Schemes, http://math.shinshu-u.ac.jp/ ${ }^{\text {hanaki/as/ }}$

[28] A. Hanaki and K. Uno, Algebraic structure of association schemes of prime order, J. Algebraic Combin., 23, (2006), 189-195.

[29] A. Herman, Trivial Schur indices for reality-based algebras with two nonreal elements, preprint, available at arXiv:1905.00445v2 [math.RA]

[30] A. Herman, M. Hirasaka, and S. Oh, Zeta functions for tensor products of locally coprime integral adjacency algebras of association schemes, Comm. Algebra, 45 (11), (2017), 4896-4905.

[31] A. Herman, M. Muzychuk, and B. Xu, Noncommutative reality-based algebras of rank 6, Comm. Algebra, 46 (1), (2018), 90-113.

[32] A. Herman, M. Muzychuk, and B. Xu, The recognition problem for table algebras and reality-based algebras, J. Algebra, 479 (2017), 173-191.

[33] A. Herman and A. Rahnamai Barghi, Schur indices of association schemes, J. Pure Appl. Algebra, (2011),

[34] A. Herman and G. Singh, Central torsion units of integral reality-based algebras with a positive degree map, Intl. Elec. J. Algebra, 21, (2017), 121-126.

[35] D.G. Higman, Coherent algebras, Linear Algebra Appl., 93 (1987), 209-239. 
[36] P. Etinghof, S. Gelaki, D. Nikshych, and V. Ostrik, Tensor Categories, Mathematical Surveys and Monographs, Vol. 205, American Mathematical Society, Providence, RI, 2015.

[37] Y. Hironaka, Zeta functions of integral group rings of metacyclic groups, Tsukuba J. Math., 5 (2), (1981), 267-283; Corrections Tskuba J. Math., 9 (2), (1985), 373-374.

[38] M. Klin and M. Ziv-Av, A non-Schurian coherent configuration on 14 points exists, Des. Codes Cryptogr., 84 (1-2), (2017), 203-221.

[39] T. Komatsu, Tamely ramified Eisenstein fields with prime power discriminants, Kyushu J. Math., 62 (1), (2008), 1-13.

[40] M. Linckelmann, The Block Theory of Finite Group Algebras, London Mathematical Society Student Texts, Vol. 91-92, Cambridge University Press, Cambridge, UK, 2018.

[41] G. Lusztig, Leading coefficients of character values of Hecke algebras, in: Proc. Sympos. Pure Math., Vol. 47, American Mathematical Society, Providence, RI, 1987, pp. 235-273, Part II.

[42] L. Solomon, Zeta functions and integral representation theory, Adv. Math., 26, (1977), 306-326.

[43] W. Martin and H. Tanaka, Commutative association schemes, European J. Combin., 30 (6), (2009), 1497-1525.

[44] M. Muzychuk, V-rings of permutation groups with invariant metric. Ph.D. thesis, Kiev State University, 1987.

[45] I. Reiner, Zeta functions of integral representations, Comm. Algebra, 8 (10), (1980), 911-925.

[46] Y. Takegahara, Zeta functions of integral group rings of abelian $(p, p)$-groups, Comm. Algebra, 15 (12), (1987), 2565-2615.

[47] P. Terwilliger, The subconstituent algebra of an association scheme I, J. Alg. Combin., 1 (1992), 363-388.

[48] J. Vidali, Using symbolic computation to prove nonexistence of distanceregular graphs, Electron. J. Combin., 25, (4), (2018), Paper 4.21, 10 pp.

[49] P. Terwilliger, The subconstituent algebra of an association scheme III, $J$. Alg. Combin., 2 (1993), 177-210. 
[50] M. Tomiyama and N. Yamazaki, The subconstituent algebra of a strongly regular graph, Kyushu J. Math., 48 (2), (1994), 323-334.

[51] B. Xu, Wedge-direct products of table algebras with applications to association schemes I, 47 (8), Comm. Algebra, (2019), 3309-3328.

[52] B. Xu, Table algebras with central fusions, Comm. Algebra, 46 (3), (2018), 1018-1031.

[53] B. Xu, On maximal actions and $\omega$-maximal actions of finite hypergroups, $J$. Algebraic Combin., 27, (2008), 127-141.

[54] P.-H. Zieschang, Theory of Association Schemes, Springer-Verlag, Berlin, 2005.

[55] P.-H. Zieschang, Algebraic Structure of Association Schemes, Lecture Notes in Mathematics No. 1628, Springer-Verlag, Berlin, 1996.

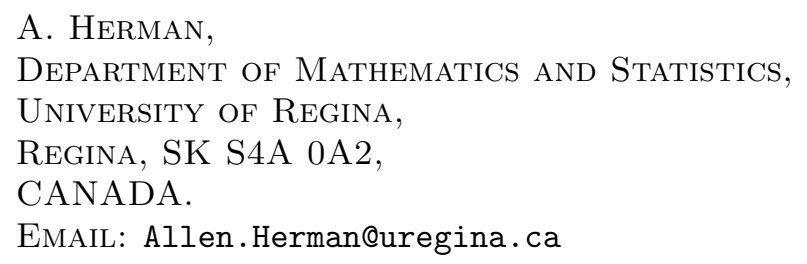

